\title{
Some New Conformal Covariants
}

\author{
V. Wünsch
}

\begin{abstract}
By means of a certain conformal covariant differentiation process explicit formulae are derived for

(i) a conformally invariant generalized Bach tensor in dimension 6

(ii) conformally invariant differential operators acting on weighted functions, especially one with leading term $\square^{4}$

(iii) conformal covariants on symmetric, trace-free p-tensor bundles, especially one with leading term $\square^{2}$

(iv) conformal covariants on differential forms.
\end{abstract}

Furthermore, theorems for uniqueness, existence and non-existence of conformal covariants, in particular in dimension 4, are given.

Keywords: Conformal transformation, conformally invariant tensor, conformally invariant differential operator, Bach tensor, conformal covariant derivative

AMS subject classification: 53 A 30,58 G 99

\section{Introduction}

The theory of conformal transformations of a pseudo-Riemannian manifold (M, g) plays a fundamental role in questions of geometry and physics. The investigation of conformally invariant differential operators and tensors on a conformal manifold is an active area of research (see, e.g., [2 - 7, 11, 12, 14, 16, 18 - 23, 25, 27, 29, 31, 34, 36, 37, 40, 41]). More generally, by a conformal covariant, we shall mean a universal polynomial expression in covariant derivatives with coefficients depending polynomially on the metric, its inverse, the curvature tensor, and its covariant derivatives which acts between conformally weighted tensor bundles and is unchanged when the metric is scaled. The Bach tensor in dimension 4 [1, 25, 37] and the conformally invariant Laplacian [43] are the most basic non- trivial examples. The polynomial conformal covariants have a great variety of applications, for example, in the representation theory of the conformal group (see, e.g., $[9,31]$ ), in the "conformal extension" of the heat equation [10], in spectral theory $[8,9]$, for Lagrangian formulation of both general relativity and conformal field theories $[1,2,16,18,40,41]$, in describing massless fields and wave propagation in curved space-times $[2,13,25,30,32,39,42]$. It is an important problem to give a survey of all conformal covariants or, with less pretension, to give a method for generating special classes of conformal covariants. Some of these procedures and conformal covariants are well known (see, e.g., $[2,5,16,19,22,40])$. The existence of a family of

V. Wünsch: Friedrich-Schiller-Universität, Math. Inst., Ernst-Abbe-Platz 1-4, D-07743 Jena 
conformal covariants on weighted functions with the leading part being a power of the Laplacian is proved in [23].

In this paper we present a relatively simple derivation of explicit formulae for some conformal covariants using a method given in [38], in particular, the notation " conformal covariant derivative" is fundamental. Most but not all invariant operators in flat space admit a curved generalization. The basic reason for the non-existence in dimension 4 is an obstructing Bach tensor expression. For example, a curved version of $\square^{3}$ in dimension $n \geq 5$ was found in [38], but there is no curved version of $\square^{3}$ in dimension 4 (see [24]). By means of our method we simplify essentially the proof of this result and give further examples for the non-existence of curved analogues of flat operators.

The paper is organized as follows. In Section 2, the basic ideas and results of [27, 38] are presented. In Section 3 we give an extension of the conformally invariant Bach tensor to dimension 6, some remarks on Euler-Lagrange tensors and state two unsolved problems. In Section 4 we investigate the general structure of conformal covariants with leading terms $\square^{k}$ acting on weighted functions by means of which one can derive explicit formulae for these operators without excessive calculation. In particular, a curved version of $\square^{4}$, two second-order conformal covariants and a proof of the nonexistence of a curved analogue of $\square^{4}$ in dimension 6 are given. Furthermore, a generating system for the set of all conformal covariants with leading term $\square^{3}$ is introduced. Conformal covariants acting on symmetric, trace-free p-tensors bundles are generated

in Section 5. In particular, we derive a fourth-order operator $\stackrel{s}{D}_{(4, p)}$ with leading term $\square^{2}$. For this operator the obstructing Bach tensor expression occuring in dimension 4 can be substituted by means of a suitable second-order operator. Finally, in Section 6 we consider conformal covariants acting on differential forms. A second-order and fourth-order conformal covariant with leading terms $\square$ and $\square^{2}$, respectively, was found by Branson [5] and the present author [40]. We present a new second-order conformal covariant and prove that there is no conformal covariant on 2-forms with leading term $\square^{2}$ in dimension 4 .

\section{Conformal covariants}

Let $(M, g)$ be a pseudo-Riemannian $C^{\infty}$ manifold of dimension $n(n \geq 4)$ and

$\begin{array}{ll}g_{a b} & \text { the local components of the covariant metric tensor } \\ g^{a b} & \text { the local components of the contravariant metric tensor } \\ \nabla_{a} & \text { the Levi Civita connection } \\ R_{a b c d} & \text { the curvature tensor } \\ R_{a b}:=R_{a \cdot c b}^{c} & \text { the Ricci tensor } \\ R & \text { the scalar curvature tensor } \\ C_{a b c d} & \text { the Weyl curvature tensor. }\end{array}$

In the following we use the sign convention according to

$$
\nabla_{[a} \nabla_{b]} T_{c}=-\frac{1}{2} R_{a b c .} T_{d}
$$


We consider linear differential operators acting on a space $\mathcal{T}$ of $C^{\infty}$ tensor fields of a certain type with (possibly) some list of symmetry conditions. Suppose that the coefficients of these operators are polynomials in $g_{a b}$ and the partial derivatives of $g_{a b}$ up to a certain order.

Let $\mathcal{D}_{k}$ be the set of all polynomial operators of order less than $k$ and $\mathcal{D}:=\bigcup_{k} \mathcal{D}_{k}$. Then obviously $\mathcal{D}$ is an algebra $[27,40]$.

An operator $D(g) \in \mathcal{D}$ is said to be of conformal weight $\omega$ if under a uniform dilatation of the metric $\bar{g}_{a b}=e^{2 \phi} g_{a b}$ with $\phi=$ const, $D(g)$ transforms according to $D(g)=e^{2 \omega \phi} D(g)$. Let $\mathcal{D}(\omega)$ be the set of all operators of conformal weight $\omega$. Then $\mathcal{D}=\oplus_{\omega} \mathcal{D}(\omega)$.

Definition 2.1. An operator $D(g) \in \mathcal{D}(\omega)$ is said to be conformally invariant of conformal weight $\left(\omega, \omega_{0}\right)$ on $\mathcal{T}$ (or shortly a conformal covariant) if a real number $\omega_{o}$ exists such that under a conformal change of the metric

$$
\bar{g}_{a b}=e^{2 \phi} g_{a b}, \quad \phi \in C^{\infty}(M)
$$

$D(g)$ transforms according to

$$
D(\bar{g})\left[e^{2 \omega_{0} \phi} u\right]=e^{2\left(\omega+\omega_{0}\right) \phi} D(g)[u]
$$

for all $u \in \mathcal{T}$.

Remark 2.1. A zeroth-order conformal covariant is called a conformally invariant tensor.

\section{Examples.}

(i) The conformal Laplacian

$$
D_{(2)}:=\nabla^{a} \nabla_{a}-\frac{n-2}{4(n-1)} R
$$

is a second-order conformal covariant on functions with $\omega=-1, \omega_{0}=\frac{1}{4}(2-n)$ [43].

(ii) The Bach tensor

$$
B_{i_{1} i_{2}}:=\nabla_{a} \nabla_{b} C_{. i_{1} i_{2} .}^{a}+\frac{n-3}{n-2} C_{. i_{1} i_{2} .}^{a}{ }^{b} R_{a b}
$$

is a conformally invariant tensor of weight $\omega=-1$ if $n=4[1,14,25,37,39]$.

If $D(g) \in \mathcal{D}(\omega)$, then under the conformal change $(2.2) D(g)$ has a transformation law of the form

$$
D(\bar{g})\left[e^{2 \omega_{0} \phi} u\right]=e^{2\left(\omega+\omega_{0}\right) \phi}\left\{D(g)+\sum_{k=1}^{m} P_{k}\left(\omega_{0}, g, \phi\right)\right\}[u] \quad(u \in \mathcal{T})
$$

where the operators $P_{k}\left(\omega_{0}, g, \phi\right)$ are $k$-homogeneous in the derivatives of $\phi$ up to a certain order $[27,40] . D(g)$ is conformally invariant if and only if the conformal variation $P_{1}\left(\omega_{0}, g, \phi\right)$ vanishes, i.e., for constructing conformal covariants it is sufficient to calculate only "to the first order in derivatives of $\phi "[27,40]$. 
Let $\vartheta(\omega)$ be the set of those elements of $\mathcal{D}(\omega)$ which contain only first order derivatives of $\phi$ in their transformation law. Furthermore, let $\vartheta:=\oplus_{\omega} \vartheta(\omega)$ and $\vartheta_{0}$ be the subset of zeroth-order operators (i.e. tensors) of $\vartheta$. Then $D(g)$ is an element of $\vartheta$ if and only if $P_{1}\left(\omega_{0}, g, \Phi\right)$ has the form

$$
P_{1}\left(\omega_{0}, g, \phi\right):=\nabla_{\gamma} \phi X^{\gamma} D
$$

Definition 2.2. The linear operator $X^{\gamma}$ defined on $\vartheta$ by $(2.7)$ is called the infinitesimal generator of $D$.

It is easy to show that $X^{\gamma}$ is a derivation, i.e. $X^{\gamma}$ obeys the Leibniz rule and commutes with contractions.

Corollary 2.1. $D \in \mathcal{D}$ is a conformal covariant if and only if $D \in \vartheta$ and $X^{\gamma} D=0$.

If $D \in \vartheta$, then we have in general $\nabla_{a} D \notin \vartheta$.

Definition 2.3. For $D \in \vartheta$ the operator

$$
\stackrel{c}{\nabla}_{a} D:=\nabla_{a} D+P_{a k} X^{k} D
$$

where

$$
P_{a b}:=\frac{1}{n-2}\left(R_{a b}-\frac{1}{2(n-1)} R g_{a b}\right)
$$

is called the conformal covariant derivative of $D$.

The following theorem holds (see [27, 40]):

\section{Therorem 2.1.}

(i) The conformal covariant derivative $\stackrel{c}{\nabla}_{a}$ a derivation.

(ii) $\stackrel{c}{\nabla}_{a}: \vartheta \rightarrow \vartheta$.

(iii) The "Ricci identity" for $\stackrel{c}{\nabla}_{a}$ has the form

$$
\stackrel{c}{\nabla}_{[a} \stackrel{c}{\nabla}_{b]} D(u):=(C, D[u])_{a b}+\frac{1}{2(n-3)} \nabla_{u} C_{a b \gamma .}{ }^{u} X^{\gamma} D[u]
$$

where $u \in \mathcal{T}$ and $(C, D[u])_{a b}$ is the term one obtains from the right-hand side of the usual Ricci identity $\nabla_{[a} \nabla_{b]} D(u):=(R, D[u])_{a b}$ by substitution of $R$ by $C$.

(iv) For $D \in \vartheta(\omega)$

$$
\left[X^{\gamma}, \stackrel{c}{\nabla} a\right] D[u]:=\left\{X^{\gamma}, \stackrel{c}{\nabla}{ }_{a}-\stackrel{c}{\nabla}_{a} X^{\gamma}\right\} D[u]=2\left(\omega+\omega_{0}\right) \delta_{a}^{\gamma} D[u]+\Delta_{a}^{\gamma} D[u]
$$

is valid where

$$
\begin{aligned}
\Delta_{a}^{\gamma}\left(T_{i j \ldots}{ }^{k m \ldots}\right)= & \Delta_{a b}^{\gamma k} T_{i j \ldots}{ }^{b m \ldots}+\Delta_{a b}^{\gamma m} T_{i j \ldots}{ }^{k b \ldots}+\ldots \\
& -\Delta_{a i}^{\gamma b} T_{b j \ldots}{ }^{k m \ldots}-\Delta_{a j}^{\gamma b} T_{i b \ldots}{ }^{k m \ldots}-\ldots
\end{aligned}
$$


and

$$
\Delta_{a i}^{k m}:=\delta_{a}^{k} \delta_{i}^{m}+\delta_{i}^{k} \delta_{a}^{m}-g_{a i} g^{k m} .
$$

(v) The algebra $\vartheta_{0}$ is generated by the tensors

$$
g^{a b}, \quad g_{a b}, \quad \stackrel{c}{\nabla}\left(i_{1} \ldots \stackrel{c}{\nabla}_{i_{r}} C_{\left.. i_{r+1} i_{r+2}\right)}^{a} . \quad(r \in \mathbb{N}) .\right.
$$

(vi) Every operator $D \in \vartheta$ can be represented in the form

$$
D=\sum_{k=0}^{m} B^{(k)} \otimes \nabla^{c}(k)
$$

where $\stackrel{c}{\nabla}^{(k)}$ denotes $\left\{\stackrel{c}{\nabla}\left(i_{1} \ldots \stackrel{c}{\nabla}_{\left.i_{k}\right)}\right\}\right.$ and $B^{(k)} \in \vartheta_{0}(k=0, \ldots, m)$.

Examples. We have (see $[27,40]$ )

$$
\begin{aligned}
& X^{\gamma} C_{a b c d}=0, \quad X^{\gamma} \nabla_{a}^{c} C_{. b c d}^{a}=(n-3) C_{. b c d}^{\gamma}, \quad \stackrel{c}{\nabla}{ }_{a} C_{b d e f}=\nabla_{a} C_{b d e f} \\
& \stackrel{c}{\nabla}{ }_{a} u=\nabla_{a} u, \quad B_{i_{1} i_{2}}=\stackrel{c}{\nabla} \stackrel{c}{\nabla}_{b} C_{. i_{1} i_{2} .}^{a}, \quad X^{\gamma} B_{i_{1} i_{2}}=2(n-4) \nabla_{a} C_{. i_{1} i_{2}}^{a} . \\
& X^{\gamma} \stackrel{c}{\nabla} a=2 \omega_{0} \delta_{a}^{\gamma} u+\Delta_{a}^{\gamma} u, \quad \stackrel{c}{\nabla} \stackrel{c}{\nabla}_{b} u=\nabla_{a} \nabla_{b} u+P_{a \gamma} X^{\gamma} \stackrel{c}{\nabla}{ }_{b} u \\
& \stackrel{c}{\square}:=\stackrel{c}{\nabla}^{a} \stackrel{c}{\nabla}_{a}=\nabla^{a} \nabla_{a}+\frac{\omega_{0}}{n-1} R \text { on } \omega_{0} \text {-weighted functions } \\
& X^{\gamma} \stackrel{c}{\square}=\left(4 \omega_{0}+n-2\right) \stackrel{c}{\nabla^{\gamma}}=0 \quad \Longleftrightarrow \quad 4 \omega_{0}=2-n .
\end{aligned}
$$

\section{On the generalization of the Bach tensor}

When Latin indices with subindices (e.g. $\left.i_{1}, \ldots, i_{r}\right)$ appear in the sequel, we assume that symmetrization has been carried out over the indices. We denote the trace-free symmetric part of a tensor $T$ by $T S(T)$. Let $\mathcal{S}_{r}(\omega, n)$ be the set of all conformally invariant, symmetric, trace-free tensors with weight $\omega$ and covariant rank $r$. There is a natural generalization $B_{i_{1} i_{2}}^{(k)} \in \mathcal{S}_{2}(\omega, n)$ of the Bach tensor $B_{i_{1} i_{2}}^{(0)}:=B_{i_{1} i_{2}}$ in dimension four to any even dimension $n$ with $\omega=\frac{2-n}{2}=-(k+1), k \in \mathbb{N}_{0}$ (see [3,19]). The tensor $B_{i_{1} i_{2}}^{(k)}$ has only the linear leading term $\square^{c}(k) B_{i_{1} i_{2}}$ (see [28]). Because of Theorem 2.1/(v) the Weyl curvature correction terms

$$
T_{i_{1} i_{2}}^{(k)}:=B_{i_{1} i_{2}}^{(k)}-\square^{c}(k) B_{i_{1} i_{2}}
$$

are generated by the tensors (2.12). Obviously, we cannot expext the uniqueness of the extension of $\square^{c}(k) B_{i_{1} i_{2}}$ to a conformally invariant tensor for $k>0$ (in the case of $k=0$ see $[37])$. 
Problem A. Find correction terms $T_{i_{1} i_{2}}^{(k)}$ for $k>0$.

Theorem 3.1. The tensor

$$
\begin{aligned}
B_{i_{1} i_{2}}^{(1)}:= & \stackrel{c}{\square} B_{i_{1} i_{2}} \\
& +\left\{\frac{2(n-4)}{(n-3)} \nabla_{a} C_{. i_{1} . .}^{a b c} \nabla_{d} C_{. i_{2} b c}^{d}-\nabla_{a} C_{. . i_{1}}^{a b c} \nabla_{d} C_{. b c i_{2}}^{d}\right\} \\
& +2 C_{i_{1} . . i_{2}}^{a b} B_{a b}
\end{aligned}
$$

is an element of $\mathcal{S}_{2}(-2,6)$.

Proof. The Ricci identity (see (2.10))

$$
\nabla_{[a} \nabla_{b]} T_{c}:=-\frac{1}{2} C_{a b c .}{ }^{d} T_{d}-\frac{1}{2(n-3)} \nabla_{u} C_{. \gamma a b}^{u} X^{\gamma} T_{c}
$$

and the Bianchi identity

$$
\nabla_{[a} C_{b c] i j}+\frac{1}{n-3}\left[g_{j[a} \nabla_{\mid u} C_{. i \mid b c]}^{u}-g_{i[a} \nabla_{\mid u} C_{. j \mid b c]}^{u}\right]=0
$$

imply

$$
\begin{aligned}
(n-3)\left[\stackrel{c}{\square} C_{b a i j}+2 \stackrel{c}{\nabla}\left[a \mid c{ }_{[a}^{\nabla}{ }_{u} C_{. \mid b] i j}^{u}-\right.\right. & \left.C_{u d b a} C_{. . i j}^{u d}-4 C_{u b[i \mid .}^{d} C_{. a \mid j] d}^{u}\right] \\
& +2 \stackrel{c}{\nabla}_{[j \mid} \stackrel{c}{\nabla}_{u} C_{. \mid i] b a}^{u}+4 g_{[i[a} B_{j] b]}=0
\end{aligned}
$$

and

$$
\begin{aligned}
\stackrel{c}{\nabla}{ }_{u} \stackrel{c}{\square} C_{. i_{1} i_{2} .}^{u}{ }^{b}= & \stackrel{c}{\square} \nabla_{u}^{c} C_{. i_{1} i_{2} .}^{u}+2 \nabla^{a} C_{a u i_{1}}^{k} C_{.\left(k i_{2}\right) .}^{u}{ }^{b} . \\
& +\nabla^{a} C_{a u . .}^{b k} C_{. i_{1} i_{2} k}^{u}+4 C_{a u i_{1} .}^{k} \nabla^{a} C_{.\left(k i_{2}\right) .}^{u}{ }^{b} \\
& +2 C_{a u . .}^{b k} \nabla^{a} C_{. i_{1} i_{2} k}^{u}+\frac{1}{n-3} \nabla_{l} C_{. k a u}^{l} X^{k}\left(\nabla^{a} C_{. i_{1} i_{2} .}^{u}{ }^{b}\right) .
\end{aligned}
$$

Hence, on account of (2.11) and (2.15),

$$
\begin{aligned}
X^{\gamma}\left(\stackrel{c}{\square} B_{i_{1} i_{2}}\right) & =\left[X^{\gamma}, \stackrel{c}{\nabla} a\right] \stackrel{c}{\nabla}{ }^{a} B_{i_{1} i_{2}}+\stackrel{c}{\nabla}\left[X^{\gamma}, \stackrel{c}{\nabla} a\right] B_{i_{1} i_{2}} \\
& =(n-10) \stackrel{c}{\nabla}{ }^{\gamma} B_{i_{1} i_{2}}+4 \stackrel{c}{\nabla}{ }_{i_{1}} B_{i_{2}}{ }^{\gamma}+\stackrel{c}{\square} X^{\gamma} B_{i_{1} i_{2}} \\
& =(n-6)\left[3 \stackrel{c}{\nabla}^{\gamma} B_{i_{1} i_{2}}-2 \stackrel{c}{\nabla}_{i_{1}} B_{i_{2}}{ }^{\gamma}\right]-X^{\gamma}\left(T_{i_{1} i_{2}}^{(1)}\right) .
\end{aligned}
$$

Consequently,

$$
X^{\gamma}\left(B_{i_{1} i_{2}}^{(1)}\right)=(n-6)\left[3 \nabla^{\gamma} B_{i_{1} i_{2}}-2 \stackrel{c}{\nabla}_{i_{1}} B_{i_{2}}^{\gamma}\right]
$$

Remark 3.1. In dimension 6 , the conformal tensor $B_{i_{1} i_{2}}^{(1)}$ has been already derived in [28]. A generating system for $\mathcal{S}_{2}(-2, n)$ is also given in [28, 39]. In [39] one can find 
applications of the tensor $B_{i_{1} i_{2}}^{(1)}$ to the theory of Huygens' principle in dimension 6 (see also [36]).

Supposing a Lagrangian $L$ to be an element of $\mathcal{S}_{0}\left(-\frac{n}{2}, n\right)$, by the action

$$
\delta \int L \sqrt{\left|\operatorname{det}\left(g_{a b}\right)\right|} d x=: \int E^{a b}(L) \delta g_{a b} \sqrt{\left|\operatorname{det}\left(g_{a b}\right)\right|} d x=0
$$

an Euler-Lagrange tensor $E_{a b}(L)$ is defined, which has the properties $[15,39]$

$$
E_{a b}(L) \in \mathcal{S}_{0}\left(-\frac{n}{2}+1, n\right), \quad E_{[a b]}(L)=0, \quad g^{a b} E_{a b}(L)=0, \quad \nabla^{a} E_{a b}(L)=0 .
$$

Example. $n=4, E_{a b}\left(C_{i j k l} C^{i j k l}\right)=c B_{a b} \quad(c \in \mathbb{R} \backslash\{0\})$ (see, e.g., [39]).

A conformal tensor $T$ is called trivial if it is generated by $\left\{g_{a b}, g^{a b}, C_{a b c d}\right\}$.

In $[28,39]$ a generating system of $\mathcal{S}_{0}(-3, n)$ was found. The only non-trivial conformal tensors from $\mathcal{S}_{0}(-3, n)$ are multiples of

$$
\begin{aligned}
S_{0}(-3, n):= & \frac{10-n}{2}\left[\nabla_{u} C_{a b c d} \nabla^{u} C^{a b c d}-\frac{4(n-2)}{(n-3)^{2}} \nabla_{u} C_{\ldots .}^{u a b c} \nabla_{v} C_{. a b c}^{v}\right] \\
& -2\left(\square-\frac{2}{n-1} R\right) C_{a b c d} C^{a b c d} .
\end{aligned}
$$

Independently, the conformal invariance of $S_{0}(-3, n)$ was verified also in [19]. The tensor $B_{i_{1} i_{2}}^{(1)}$ given by $(3.1)$ is not an Euler-Lagrange tensor, however in six dimensions an Euler-Lagrange tensor $E_{a b}\left(S_{0}(-3, n)\right)$ is a linear combination of $B_{i_{1} i_{2}}^{(1)}$ and further conformal tensors of $\mathcal{S}_{0}(-2,6)$ (see [39]).

Problem B. Find non-trivial conformal tensors of $\mathcal{S}_{0}(\omega, n)$ if $\omega<-3$.

Conjecture A. For every even $n(n \geq 4)$ there is an conformal Euler-Lagrange tensor $W_{i_{1} i_{2}}^{(k)} \in \mathcal{S}_{0}\left(-\frac{n}{2}+1, n\right)\left(k=\frac{n-4}{2} \in \mathbb{N}_{0}\right)$ with the linear leading term $\square^{k} B_{i_{1} i_{2}}$.

\section{Conformal covariants acting on weighted functions}

Let $\mathcal{T}_{0}$ be the set of all $C^{\infty}$ scalar $\omega_{0}$-weighted functions and $\square=\nabla^{a} \nabla_{a}, \stackrel{c}{\square}=\stackrel{c}{\nabla}^{a} \stackrel{c}{\nabla}_{a}$. The following theorem was proved in [23].

Theorem 4.1. If $n$ is odd, then there is for each $k \geq 1$ a conformal covariant $D_{(2 k)}$ of order $(2 k)$ with $\omega=-k, 4 \omega_{0}=2 k-n$ and leading term $\square^{k}$. If $n$ is even, then the same result is true with the restriction $1 \leq k \leq \frac{n}{2}$.

Using the notions and results of Section 2 here we analyze the general structure of $D_{(2 k)}$ and present an algorithm for an explicit construction of $D_{(2 k)}$, which simplifies those given in [23]. Relation (2.11) implies

$$
\begin{aligned}
X^{\gamma} \stackrel{c}{\square}^{k} & =\left[X^{\gamma}, \stackrel{c}{\nabla}_{a}\right] \nabla^{c} a \stackrel{c}{\square} k-1+\stackrel{c}{\nabla}^{a}\left[X^{\gamma}, \stackrel{c}{\nabla} a\right] \stackrel{c}{\square}^{k-1} \\
& =b_{k} \stackrel{c}{\nabla}^{\gamma} \stackrel{c}{\square}^{k-1}+\stackrel{c}{\square} X^{\gamma} \stackrel{c}{\square}^{k-1}
\end{aligned}
$$


where $b_{k}:=4 \omega_{0}-4 k+n+2$. Hence, by induction,

$$
\begin{aligned}
X^{\gamma} \stackrel{c}{\square}^{k}= & b_{k} \stackrel{c}{\nabla}^{\gamma} \stackrel{c}{\square}^{k-1}+b_{k-1} \stackrel{c}{\square}^{c} \nabla^{\gamma} \stackrel{c}{\square}^{k-2}+\ldots+b_{1} \stackrel{c}{\square}^{k-1} \stackrel{c}{\nabla}^{\gamma} \\
= & a_{k} \stackrel{c}{\nabla}^{\gamma} \stackrel{c}{\square}^{k-1}+a_{k-1}\left[\stackrel{c}{\square}, \stackrel{c}{\nabla}^{\gamma}\right] \stackrel{c}{\square}^{k-2} \\
& +a_{k-2} \stackrel{c}{\square}\left[\stackrel{c}{\square}, \stackrel{c}{\nabla}^{\gamma}\right] \square^{k-3}+\ldots+a_{1} \stackrel{c}{\square}^{k-2}[\stackrel{c}{\square}, \stackrel{c}{\nabla} \gamma]
\end{aligned}
$$

where $[\stackrel{c}{\square}, \stackrel{c}{\nabla} \gamma]:=\stackrel{c}{\square} \stackrel{c}{\nabla}^{\gamma}-\stackrel{c}{\nabla}^{\gamma} \stackrel{c}{\square}$ and

$$
a_{m}=\sum_{r=1}^{m} b_{r}=m\left(4 \omega_{0}+n-2 m\right) \quad(m=1, \ldots, k) .
$$

We obtain by means of (2.10)

$$
\left[\stackrel{c}{\square}, \nabla^{\gamma}\right] \stackrel{c}{\square}^{m}=\frac{1}{n-3}\left[B_{. a}^{\gamma}-2\left(\nabla_{u} C_{. a}^{u . b \gamma}\right) \stackrel{c}{\nabla}_{b}\right] X^{a} \stackrel{c}{\square}^{m}=: \frac{1}{n-3} F_{. a}^{\gamma} X^{a} \stackrel{c}{\square}^{m}
$$

if $m \geq 1$ and $\left[\stackrel{c}{\square}, \stackrel{c}{\nabla}^{\gamma}\right] \stackrel{c}{\square} m=0$ if $m=0$.

Choosing a flat metric as a test metric we get from (4.1)

Lemma 4.1. Necessary for $D_{(2 k)}$ to be a conformal covariant is

$$
a_{k}=k\left(4 \omega_{0}+n-2 k\right)=0 .
$$

In the case $4 \omega_{0}=2 k-n$ (see Lemma 4.1$)$ one has

$$
a_{m}=2 m(k-m) \quad(m=1, \ldots, k) .
$$

Thus we have proved

Lemma 4.2. For every $k \geq 1$ and $4 \omega_{0}=2 k-n$ there holds

$$
X^{\gamma} \stackrel{c}{\square}^{k}= \begin{cases}2 \sum_{m=2}^{k-1} m(k-m) \stackrel{c}{\square^{k-m-1}}\left[\stackrel{c}{\square}, \stackrel{c}{\nabla}^{\gamma}\right] \stackrel{c}{\square}^{m-1} & \text { if } k \geq 3 \\ 0 & \text { if } k \leq 2 .\end{cases}
$$

Now from (2.8), Theorem 2.1 and $\stackrel{c}{\nabla}_{a} B_{. b}^{a}=0$ we imply immediately

Lemma 4.3. The coefficients of all Weyl curvature correction terms of $D_{(2 k)}-\square^{k}$ which are linear with respect to the conformal covariant derivatives of the Weyl tensor are up to real numbers conformal covariant derivatives of the Bach tensor of order $p$ with $0 \leq p \leq k-2$.

Conjecture B. For every $n$ and $k$ with $2 k \leq n$ and $n \geq 4$ there is a conformal covariant $D_{(2 k)}$, which reduces to $\square^{k}$ for an Einstein metric.

\section{Examples.}

(i) The operator

$$
D_{(2)} \equiv \stackrel{c}{\square}=\square-\frac{n-2}{4(n-1)} R
$$


is the only second order conformal covariant on $\mathcal{T}_{0}$ with $\omega=-1, \omega_{0}=2-n$ and leading term $\square$ (see, e.g., $[40,43]$ ).

(ii) If $k=2$, then we get $X^{\gamma} \square^{c}=0$ from Lemma 4.2. Consequently, a fourthorder conformal covariant on $\mathcal{T}_{0}$ with $\omega=-2,4 \omega_{0}=4-n$ and leading term $\square^{2}$ has the form

$$
D_{(4)}:=\stackrel{c}{\square}^{2}+c_{0} C_{a b c d} C^{a b c d} \quad\left(c_{0}=\text { const }\right) .
$$

The operator $\square^{c}$ is exactly that conformal covariant which S. Paneitz introduced in 1983 [33]. The proof of the uniqueness is very easy (see, e.g., [40]).

(iii) If $k=3$, then because of

$$
\left.\begin{array}{l}
X^{\gamma} \stackrel{c}{\square}=\left(4 \omega_{0}+n-2\right) \stackrel{c}{\nabla^{\gamma}}=4 \stackrel{c}{\nabla} \gamma \\
X^{\gamma} T S\left(\stackrel{c}{\nabla}_{i_{1}} \stackrel{c}{\nabla}_{i_{2}}\right)=(4-n) T S\left(\delta_{i_{1}}^{\gamma} \stackrel{c}{\nabla}_{i_{2}}\right)
\end{array}\right\}
$$

we have

$$
\begin{aligned}
X^{\gamma}\left(\stackrel{c}{\square}^{3}\right) & =4[\stackrel{c}{\square}, \stackrel{c}{\nabla}]] \stackrel{c}{\square} \\
& =\frac{16}{n-3}\left[B^{\gamma a}-2 \stackrel{c}{\nabla}_{u} C^{u a b \gamma} \stackrel{c}{\nabla}_{b}\right] \stackrel{c}{\nabla} a \\
& =\frac{16}{n-3}\left[B^{\gamma a}-\frac{1}{n-4} X^{\gamma}\left(B^{a b}\right) \stackrel{c}{\nabla_{b}}\right] \stackrel{c}{\nabla}_{a} \\
& =-\frac{16}{(n-3)(n-4)} X^{\gamma}\left(B^{a b} \stackrel{c}{\nabla}_{b} \stackrel{c}{\nabla}_{a}\right) .
\end{aligned}
$$

Hence, the sixth-order operator found by the present author in [40]

$$
D_{(6)}^{0}:=\sqcup^{c} 3+\frac{16}{(n-3)(n-4)} B^{a b} \stackrel{c}{\nabla}_{a}^{c} \nabla_{b}
$$

is for $n>4$ a conformal covariant on $\mathcal{T}_{0}$ with $\omega=-3,4 \omega_{0}=6-n$ and leading term $\square^{3}$. If $n=4$, then $B^{a b} \nabla_{a} \nabla_{b}$ is a second-order conformal covariant on $\mathcal{T}_{0}$. On account of Lemma 4.3 it is easy to see that $c B^{a b} \nabla_{a} \nabla_{b}$ with $c=$ const is the only possible curvature correction term of $D_{(6)}^{0}$ with a linear coefficient. As a conclusion, there is no operator $D_{(6)}$ such that

$$
X^{\gamma}\left(D_{(6)}-\stackrel{c}{\square}^{3}\right)=-16\left[B^{\gamma a}-2 \stackrel{c}{\nabla}{ }_{u} C^{u a b \gamma} \stackrel{c}{\nabla}_{b}\right] \stackrel{c}{\nabla} a
$$

in dimension 4 . Thus, by means of the method given in Section 2, we obtain a simple proof of the following theorem due to Graham [24].

Theorem 4.1. If $n=4$, then there is no conformal covariant on $\mathcal{T}_{0}$ with the leading term $\square^{3}$.

Theorem 4.2. The following operators are second-order conformal covariants on $\mathcal{T}_{0}$ with $\omega=-3$ and $4 \omega_{0}=6-n(n \geq 4)$ :

$$
\begin{aligned}
D_{(2)}^{1}= & \nabla_{a}^{c}\left[\left\{C_{. d e f}^{a} C^{b d e f}-\frac{1}{4} g^{a b} C^{g d e f} C_{g d e f}\right\} \nabla_{b}\right] \\
& +\frac{(n-4)(n-6)}{4(n-3)^{2}} \nabla_{a} C^{a d e f} \nabla_{b} C_{. d e f}^{b} \\
D_{(2)}^{2}= & 2(n-10) \nabla_{a}^{c}\left[C^{b d e f} C_{b d e f} \nabla^{a}\right]+(n-6) \stackrel{c}{\square}\left[C_{a b d e} C^{a b d e}\right] .
\end{aligned}
$$


Proof. From (2.11) and (4.9), it follows after a straight computation

$$
\begin{aligned}
X^{\gamma}\left\{\nabla_{a}^{c}\left[\left\{C_{. d e f}^{a} C^{b d e f}-\frac{1}{4} g^{a b} C^{g d e f} C_{g d e f}\right\} \nabla_{b}\right]\right\} \\
=-\frac{(n-4)(n-6)}{2(n-3)} \nabla_{a} C^{a d e f} C_{. d e f}^{\gamma} \\
=-\frac{(n-4)(n-6)}{2(n-3)^{2}} X^{\gamma}\left[\nabla_{a} C_{. d e f}^{a} \nabla_{b} C^{b d e f}\right],
\end{aligned}
$$

hence $X^{\gamma} D_{(2)}^{1}=0$. The proof of $X^{\gamma} D_{(2)}^{2}=0$ is analogous

Corollary 4.1. Every sixth-order conformal covariant on $\mathcal{T}_{0}$ with $\omega=-3,4 \omega_{0}=$ $6-n(n>4)$ and leading term $\square^{3}$ has the form

$$
D_{(6)}=D_{(6)}^{0}+c_{1} D_{(2)}^{1}+c_{2} D_{(2)}^{2}+S
$$

with

$$
S=d_{1} S_{0}(-3, n)+d_{2} C^{a e b f} C_{a c d f} C_{e . . b}^{c d}+d_{3} C_{. . c d}^{a b} C_{. . e f}^{c d} C_{. . a b}^{e f}
$$

were $c_{1}, c_{2} \in \mathbb{R}$ and $d_{1}, d_{2}, d_{3} \in \mathbb{R}$.

Proof. It is easy to see that the operator $D_{(6)}^{0}-D_{(6)}$ can only contain the (linear independent) curvature monomials from $D_{(2)}^{1}, D_{(2)}^{2}$ and $S$. Now, from Lemma 4.3, Theorem 4.2 and the fact that $\mathcal{S}_{0}(-3, n)$ is generated by the monomials of $(4.15)$ (see [18, $28,39])$ the assertion follows

Theorem 4.3. The following operator is for $n \geq 5, n \neq 6$ a conformal covariant on $\mathcal{T}_{0}$ with $\omega=-4,4 \omega_{0}=8-n$ and leading term $\square^{4}$ :

$$
\begin{aligned}
& D_{(8)}:=\stackrel{c}{\square}^{4} \\
& +\frac{96}{(n-3)(n-4)}\left[B^{a b} \stackrel{c}{\nabla} \stackrel{c}{\nabla}_{b} \stackrel{c}{\square}+\left(\stackrel{c}{\nabla^{a}} B^{b d}\right) \stackrel{c}{\nabla} \stackrel{c}{\nabla}_{b} \stackrel{c}{\nabla}_{d}\right] \\
& +\frac{48}{(n-3)(n-6)}\left(\stackrel{c}{\square} B^{a b}\right) \stackrel{c}{\nabla} \stackrel{c}{a}_{b} \\
& -\frac{192}{(n-3)^{2}(n-6)}\left[\stackrel{c}{\nabla}{ }_{u} C_{\ldots .}^{u e d a} \stackrel{c}{\nabla}_{\nu} C_{. e d .}^{\nu}{ }^{b}-\stackrel{c}{\nabla}_{u} C_{\ldots .}^{u a e d}{ }^{c}{ }_{\nu} C_{. . e d}^{\nu b}\right] \nabla_{a}^{c} \stackrel{c}{\nabla}_{b} \\
& -\frac{96(n-8)}{(n-3)(n-4)(n-6)} C_{\ldots .}^{a e d b} B_{e d} \stackrel{c}{\nabla} \stackrel{c}{a}_{b}-\frac{96}{(n-3)^{2}} B^{b d}\left(\stackrel{c}{\nabla}{ }_{u} C_{. b d}^{u}{ }_{.}^{a}\right) \stackrel{c}{\nabla} \text { a } \\
& -\frac{24(n-2)(n-8)}{(n-3)^{2}(n-4)^{2}} B^{a b} B_{a b} \text {. }
\end{aligned}
$$

Proof. Supposing (4.4), i.e. $4 \omega_{0}=8-n$, from Lemma 4.2 and (4.3), (4.5) there follows

$$
\begin{aligned}
X^{\gamma} \stackrel{c}{\square}^{4} & =8 \stackrel{c}{\square}\left[\stackrel{c}{\square}, \stackrel{c}{\nabla}^{\gamma}\right] \stackrel{c}{\square}+6\left[\stackrel{c}{\square}, \stackrel{c}{\nabla}^{\gamma}\right] \stackrel{c}{\square}^{2} \\
& =\frac{2}{n-3}\left[4 \stackrel{c}{\square}\left(F_{. a}^{\gamma} X^{a} \stackrel{c}{\square}\right)+3 F_{. a}^{\gamma} X^{a} \stackrel{c}{\square}^{2}\right] \\
& =\frac{48}{n-3}\left[\stackrel{c}{\square}\left(F^{\gamma a} \stackrel{c}{\nabla}_{a}\right)+F^{\gamma a} \stackrel{c}{\nabla}_{a} \stackrel{c}{\square}\right] .
\end{aligned}
$$


Now, we have to compute the action of $X^{\gamma}$ on all Weyl curvature correction terms of $D_{(8)}$, using relation $(2.11)$. For example, we obtain

$$
\begin{aligned}
& X^{\gamma}\left(B^{a b} \stackrel{c}{\nabla} \underset{a}{\nabla_{b}} \stackrel{c}{\square}\right) \\
& =X^{\gamma}\left(B^{a b}\right) \stackrel{c}{\nabla} \stackrel{c}{\nabla}_{b} \stackrel{c}{\square}+B^{a b}\left[\left[X^{\gamma}, \stackrel{c}{\nabla} a\right] \stackrel{c}{\nabla_{b}} \stackrel{c}{\square}+\stackrel{c}{\nabla}_{a}\left[X^{\gamma}, \stackrel{c}{\nabla}, \stackrel{c}{\square}+\stackrel{c}{\nabla}_{a} \stackrel{c}{\nabla}_{b} X^{\gamma} \stackrel{c}{\square}\right]\right. \\
& =2(n-4) \nabla_{u} C^{u(a b) \gamma} \stackrel{c}{\nabla}_{a} \stackrel{c}{\nabla_{b}} \stackrel{c}{\square}+(2-n) B^{\gamma a} \stackrel{c}{\nabla}_{a} \stackrel{c}{\square}+6 B^{a b} \stackrel{c}{\nabla}_{a} \stackrel{c}{\nabla_{b}} \stackrel{c}{\nabla} \text {. }
\end{aligned}
$$

Substituting $\stackrel{c}{\square} B_{a b}$ by $B_{a b}^{(1)}$ (see (3.1)) and using (3.6), one finds

$$
X^{\gamma}\left(B_{a b}^{(1)} \stackrel{c}{\nabla} a \stackrel{c}{\nabla} b\right)=(n-6)\left[3 \stackrel{c}{\nabla}^{\gamma} B^{a b}-2 \nabla^{(a} B^{b) \gamma}\right] \stackrel{c}{\nabla}_{a} \stackrel{c}{\nabla}_{b}+(6-n) B_{a}^{(1) \gamma} \stackrel{c}{\nabla}^{a}
$$

In an analogous manner one computes the other actions. Using successively identity (2.10), for example

$$
\begin{aligned}
T S[ & \left.\stackrel{c}{\square} \stackrel{c}{\nabla}_{i_{1}} \stackrel{c}{\nabla} i_{2}-\nabla_{i_{1}}^{c} \stackrel{c}{\square} \nabla_{i_{2}}^{c}\right] \\
& =\frac{2(5-n)}{n-3} \nabla^{a} C_{a i_{1} i_{2}} \stackrel{k}{\nabla_{k}}-2 C_{. i_{1} i_{2} .}^{a}{ }^{k}{ }_{a}^{c} \stackrel{c}{\nabla} k-\frac{n-8}{2(n-3)} B_{i_{1} i_{2}},
\end{aligned}
$$

one verifies after length straight computations the assertion $X^{\gamma} D_{(8)}=0$

\section{Corollary 4.2.}

(i) If $n=6$, then

$$
B_{a b}^{(1)} \stackrel{c}{\nabla}^{a} \nabla^{c}
$$

is a second-order conformal covariant on $\mathcal{T}_{0}$ with $\omega=-4$ and $\omega_{0}=\frac{1}{2}($ see $(3.1))$.

(ii) If $n=6$, then there is no conformal covariant $D_{(8)}$ on $\mathcal{T}_{0}$ with leading term $\square^{4}$.

Proof. Assertion (i) follows multiplying $D_{(8)}$ by $(n-6)$, using (3.1). In order to prove assertion (ii) we remember that by Lemma 4.3 the linear coefficients of the correction terms of any operator $D_{(8)}^{*}$ are conformal covariant derivatives up to second order of the Bach tensor. It is easy to see that the operator $D_{(8)}$ of Theorem 4.3 contains all possible conformal covariant derivatives of $B_{a b}$ (including the zeroth order derivatives). The real coefficients of all monomials of $D_{(8)}$ are determined uniquely. In particular, the term

$$
48[(n-3)(n-6)]^{-1}\left(\stackrel{c}{\square} B^{a b}\right) \stackrel{c}{\nabla} \stackrel{c}{\nabla}_{b}
$$

cannot be substituted by other "linear" terms. Consequently, we obtain assertion (ii)

Conjecture C. There is no conformal covariant on $\mathcal{T}_{0}$ with leading term $\square^{k}$ in even dimension $n$ with $n<2 k$ and $k \geq 2$. 


\section{Conformal covariants acting on symmetric, trace-free tensor fields}

Let $\stackrel{s}{\mathcal{T}}$ pe the space of all symmetric, trace-free $C^{\infty}$-tensor fields of covariant rank $p$. In [40] the following theorem was proved.

Theorem 5.1. The operator $\stackrel{s}{D}_{(2, p)}$ defined on $\stackrel{s}{\mathcal{T}}_{p}$ by

$$
\begin{aligned}
& (\stackrel{s}{D}(2, p)[u])_{i_{1} \ldots i_{p}}:=\stackrel{c}{\square} u_{i_{1} \ldots i_{p}}-\frac{4 p}{n+2 p-2} \stackrel{c}{\nabla}_{i_{1}} \stackrel{c}{\nabla}^{k} u_{k i_{2} \ldots i_{p}} \\
& +\frac{4 p(p-1)}{(n+2 p-2)(n+2 p-4)} g_{i_{1} i_{2}} \nabla^{c} \stackrel{c}{\nabla}^{m} u_{k m i_{3} \ldots i_{p}}
\end{aligned}
$$

is a second-order conformal covariant with $\omega=-1$ and $4 \omega_{0}=2+2 p-n(n \geq 4)$.

Remark 5.1. Explicitly, we have

$$
\begin{aligned}
& \stackrel{c}{\square} u_{i_{1} \ldots i_{p}}=\left(\square-\frac{n-2}{4(n-1)} R\right) u_{i_{1} \ldots i_{p}} \\
& \stackrel{c}{\nabla_{i_{1}}} \stackrel{c}{\nabla}^{k} u_{k i_{1} \ldots i_{p}}=\nabla_{i_{1}} \nabla^{k} u_{k i_{2} \ldots i_{p}}+\frac{n+2 p-p}{2} P_{i_{1}}^{k} u_{k i_{2} \ldots i_{p}} .
\end{aligned}
$$

Theorem 5.2. If $n+2 p>6$, the operator $\stackrel{s}{D}_{(4, p)}$ defined on $\stackrel{s}{\mathcal{T}}_{p}$ by

$$
\begin{aligned}
& (\stackrel{s}{D}(4, p)[u])_{i_{1} \ldots i_{p}} \\
& :=T S\left[\stackrel{c}{\square}{ }^{2} u_{i_{1} \ldots i_{p}}+a_{1} \stackrel{c}{\square} \stackrel{c}{\nabla}_{i_{1}} \stackrel{c}{\nabla}^{a} u_{a i_{2} \ldots i_{p}}+a_{2} \stackrel{c}{\nabla} i_{i_{1}} \stackrel{c}{\nabla} i_{i_{2}} \stackrel{c}{\nabla}{ }^{a} \stackrel{c}{\nabla}{ }^{b} u_{a b i_{3} \ldots i_{p}}\right. \\
& +C_{. i_{1} i_{2} \cdot}^{a}{ }^{b}\left\{a_{3} \stackrel{c}{\square} u_{a b i_{3} \ldots i_{p}}+a_{4} \stackrel{c}{\nabla} \stackrel{c}{\nabla}^{k} u_{k b i_{3} \ldots i_{p}}\right\}+a_{5} C_{i_{1} \ldots}^{a b k} \stackrel{c}{\nabla}_{i_{2}} \stackrel{c}{\nabla}{ }_{k} u_{a b i_{3} \ldots i_{p}} \\
& +\stackrel{c}{\nabla}{ }_{u} C_{\ldots}^{u a b}\left\{a_{6} \stackrel{c}{\nabla}{ }_{a} u_{b i_{2} \ldots i_{p}}+a_{7} \stackrel{c}{\nabla}{ }_{b} u_{a i_{3} \ldots i_{p}}+a_{8} \stackrel{c}{\nabla} i_{2} u_{a b i_{3} \ldots i_{p}}\right\} \\
& \left.+a_{9} \stackrel{c}{\nabla}{ }_{u} C_{. i_{1} i_{2} .}^{u}{ }^{a} \stackrel{c}{\nabla} u_{k a i_{3} \ldots i_{p}}+a_{10} B_{i_{1}}^{a} u_{a i_{2} \ldots i_{p}}\right]
\end{aligned}
$$

with

$$
\left.\begin{array}{l}
a_{1}=\frac{-8 p}{n+2 p} \\
a_{2}=\frac{-16 p(p-1)}{(n+2 p)(n+2 p-2)} \\
a_{3}=-\frac{1}{2} a_{5}=\frac{-4 p(p-1)}{n+2 p-6}
\end{array}\right\}
$$




$$
\begin{aligned}
a_{4} & =\frac{-8 p(p-1)(n+2 p-12)}{(n+2 p)(n+2 p-6)} \\
a_{6} & =\frac{4 p(n-2 p-2)}{(n-3)(n+2 p-6)} \\
a_{7} & =\frac{a_{8}}{(1-p)}=\frac{-4 p(n-4)}{(n-3)(n+2 p-6)} \\
a_{9} & =\frac{-4 p(p-1)\left(n^{2}+2 n p-12 n+24\right)}{(n-3)(n+2 p)(n+2 p-6)} \\
a_{10} & =\frac{2 p(n-6)}{(n-3)(n+2 p-6)}
\end{aligned}
$$

is a fourth-order conformal covariant with $\omega=-2$ and $4 \omega_{0}=4+2 p-n$.

Proof. In the following the relation $T_{1} \stackrel{*}{=} T_{2}$ means $T S\left(T_{1}-T_{2}\right)=0$. From $(2.11)$ there follows (see also [35] and [40: p. 277])

$$
\begin{aligned}
& X^{\gamma}\left(\stackrel{c}{\square}^{2} u_{i_{1} \ldots i_{p}}\right) \\
& \left.\stackrel{*}{=}\left(\left[X^{\gamma}, \stackrel{c}{\nabla} a\right] \stackrel{c}{\nabla}^{a}+\stackrel{c}{\nabla^{a}}\left[X^{\gamma}, \stackrel{c}{\nabla} a\right]\right) \stackrel{c}{\square}+\stackrel{c}{\square}\left(\left[X^{\gamma}, \stackrel{c}{\nabla}{ }_{a}\right] \stackrel{c}{\nabla^{a}}+\stackrel{c}{\nabla^{a}}\left[X^{\gamma}, \stackrel{c}{\nabla} a\right]\right)\right\} u_{i_{1} \ldots i_{p}} \\
& =2\left(4 \omega_{0}-2 p+n-4\right) \stackrel{c}{\nabla} \stackrel{c}{\square}^{c} u_{i_{1} \ldots i_{p}}-4 p \stackrel{c}{\square}\left(\delta_{i_{1}}^{\gamma} \stackrel{c}{\nabla}^{d} u_{d i_{2} \ldots i_{p}}-\stackrel{c}{\nabla}_{i_{1}} u_{._{2} \ldots i_{p}}^{\gamma}\right) \\
& +\left(4 \omega_{0}-2 p+n-2\right)\left\{\stackrel{c}{\square} \stackrel{c}{\nabla}^{\gamma}-\stackrel{c}{\nabla}^{\gamma} \stackrel{c}{\square}\right\} u_{i_{1} \ldots i_{p}}-2 p\left\{\stackrel{c}{\square} \stackrel{c}{\nabla}_{i_{1}}-\stackrel{c}{\nabla}_{i_{1}} \stackrel{c}{\square}\right\} u_{. i_{2} \ldots i_{p}}^{\gamma} \\
& +2 p \delta_{i_{1}}^{\gamma}\left\{\stackrel{c}{\square} \stackrel{c}{\nabla}^{d}-\stackrel{c}{\nabla}^{d} \stackrel{c}{\square}\right\} u_{d i_{2} \ldots i_{p}}, \\
& X^{\gamma}\left(\stackrel{c}{\square} \stackrel{c}{\nabla}_{i_{1}} \stackrel{c}{\nabla}_{a} u_{. i_{2} \ldots i_{p}}^{a}\right) \\
& \stackrel{*}{=}\left(\left[X^{\gamma}, \stackrel{c}{\nabla}{ }_{a}\right] \stackrel{c}{\nabla}^{a}+\stackrel{c}{\nabla}\left[X^{\gamma}, \stackrel{c}{\nabla}{ }_{a}\right]\right) \stackrel{c}{\nabla} i_{i_{1}} \stackrel{c}{\nabla}^{d} u_{d i_{2} \ldots i_{p}}+\stackrel{c}{\square} X^{\gamma} \stackrel{c}{\nabla}_{i_{1}} \stackrel{c}{\nabla}^{k} u_{k i_{2} \ldots i_{p}} \\
& \stackrel{*}{=}\left(2 \omega_{0}-p-1\right) \delta_{i_{1}}^{\gamma} \stackrel{c}{\square} \nabla^{c} u_{k i_{2} \ldots i_{p}} \\
& +\left(2 \omega_{0}+n-2\right) \stackrel{c}{\square} \stackrel{c}{\nabla}_{i_{1}} u_{._{2} \ldots i_{p}}^{\gamma}-2(p-1) \delta_{i_{1}}^{\gamma} \stackrel{c}{\nabla} i_{2} \stackrel{c}{\nabla}_{a} \stackrel{c}{\nabla} u_{b}^{a b} u_{. . i_{3} \ldots i_{p}}^{a b} \\
& +2(p-1) \stackrel{c}{\nabla}{ }_{i_{1}} \stackrel{c}{\nabla}_{i_{2}} \stackrel{c}{\nabla}^{k} u_{k . i_{3} \ldots i_{p}}^{\gamma}+\left(4 \omega_{0}-2 p+n-4\right) \stackrel{c}{\nabla^{\gamma}} \stackrel{c}{\nabla}_{i_{1}} \stackrel{c}{\nabla}_{k} u_{._{2} \ldots i_{p}}^{k} \\
& -4(p-1) \delta_{i_{1}}^{\gamma} \stackrel{c}{\nabla}\left[\stackrel{c}{\nabla}{ }_{\left.i_{2}\right]} \stackrel{c}{\nabla}{ }_{k} u_{. . i_{3} \ldots i_{p}}^{k l}-2\left(\stackrel{c}{\nabla}{ }^{\gamma} \stackrel{c}{\nabla}_{i_{1}}-\stackrel{c}{\nabla}{ }_{i_{1}} \stackrel{c}{\nabla} \gamma\right) \stackrel{c}{\nabla}{ }_{k} u_{. i_{2} \ldots i_{p}}^{k} .\right.
\end{aligned}
$$

and

$$
\begin{aligned}
& X^{\gamma}\left(\stackrel{c}{\nabla_{i_{1}}} \stackrel{c}{\nabla} i_{i_{2}} \stackrel{c}{\nabla} \stackrel{c}{\nabla}_{b} u_{. . i_{3} \ldots i_{p}}^{a b}\right) \\
& \quad \stackrel{*}{=}\left(4 \omega_{0}-4 p+n-2\right) \delta_{i_{1}}^{\gamma} \stackrel{c}{\nabla} i_{i_{2}} \stackrel{c}{\nabla} \stackrel{c}{a}_{b} u_{. . i_{3} \ldots i_{p}}^{a b}+\left(4 \omega_{0}+2 p+n-6\right) \stackrel{c}{\nabla_{i_{1}}} \stackrel{c}{\nabla_{i_{2}}} \stackrel{c}{\nabla_{k}} u_{. . i_{3} \ldots i_{p}}^{k \gamma} .
\end{aligned}
$$

Now we have to compute the action of $X^{\gamma}$ on all Weyl curvature correction terms of 
$\stackrel{s}{D}_{(4, p)}$, using relation $(2.11)$. For instance, we obtain

$$
\begin{aligned}
& X^{\gamma}\left(C_{. i_{1} i_{2} .}^{a} \stackrel{b}{\square} u_{a b i_{3} \ldots i_{p}}\right) \\
& \stackrel{*}{=} C_{. i_{1} i_{2} \cdot}^{a}{ }^{b}\left[\left(4 \omega_{0}-2 p+n-2\right) \stackrel{c}{\nabla}{ }^{\gamma} u_{a b i_{3} \ldots i_{p}}-4 \delta_{a}^{\gamma} \stackrel{c}{\nabla}_{k} u_{. b i_{3} \ldots i_{p}}^{k}\right. \\
& \left.\quad+4 \stackrel{c}{\nabla}{ }_{a} u_{b . i_{3} \ldots i_{p}}^{\gamma}-2(p-2) \delta_{i_{3}}^{\gamma} \stackrel{c}{\nabla}_{k} u_{. a b i_{4} \ldots i_{p}}^{k}+2(p-2) \stackrel{c}{\nabla}_{i_{3}} u_{. a b i_{4} \ldots i_{p}}^{\gamma}\right] .
\end{aligned}
$$

In an analogous manner one computes the other actions. Using $4 \omega_{0}+n-2 p-4=0$ and successively identity (2.10), for instance

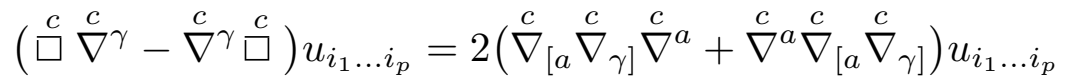

$$
\begin{aligned}
& =-2 p C_{\gamma . . i_{i}}^{a b} \stackrel{c}{\nabla}{ }_{a} u_{b i_{2} \ldots i_{p}}+\frac{p(n-2)}{n-3} \nabla_{u}^{c} C_{. \gamma a i_{1}}^{u} u_{. i_{2} \ldots i_{p}}^{a},
\end{aligned}
$$

one verifies after lengthy straight computations the assertion $X^{\gamma} \stackrel{s}{D}(4, p)=0$

Theorem 5.3. If $n+2 p>6$, the operator $\stackrel{s}{D_{(2, p)}^{*}}$ defined on $\stackrel{s}{\mathcal{T}}$ by

$$
\begin{aligned}
& \left(\stackrel{s}{D_{(2, p)}^{*}}[u]\right)_{i_{1} \ldots i_{p}}:=T S\left[C_{i_{1} \ldots}^{a b d}\left(\stackrel{c}{\nabla}{ }_{a} \stackrel{c}{\nabla} u_{b i_{2} \ldots i_{p}}-2 b_{1} \stackrel{c}{\nabla}_{i_{2}} \stackrel{c}{\nabla} u_{d a b i_{3} \ldots i_{p}}\right)\right. \\
& +b_{1} C_{. i_{1} i_{2}}^{a}{ }^{b}\left(\stackrel{c}{\square} u_{a b i_{3} \ldots i_{p}}-2 \stackrel{c}{\nabla}_{a} \stackrel{c}{\nabla}^{k} u_{k b i_{3} \ldots i_{p}}\right) \\
& +\stackrel{c}{\nabla}{ }_{u} C_{\ldots i_{1}}^{u a b}\left(b_{2} \stackrel{c}{\nabla} i_{2} u_{a b i_{3} \ldots i_{p}}+b_{3} \stackrel{c}{\nabla} a u_{b i_{2} \ldots i_{p}}+b_{4} \stackrel{c}{\nabla} u_{a i_{2} \ldots i_{p}}\right) \\
& \left.+b_{2} \stackrel{c}{\nabla}{ }_{u} C_{. i_{1} i_{2} .}^{u}{ }^{a} \stackrel{c}{\nabla}^{k} u_{k a i_{3} \ldots i_{p}}+b_{5} B_{i_{1}}^{a} u_{a i_{2} \ldots i_{p}}\right]
\end{aligned}
$$

with

$$
\left.\begin{array}{l}
\beta_{0}=(n+2 p-6)^{-1} \\
\beta_{1}=[4(n-3)]^{-1} \beta_{0}
\end{array}\right\}
$$

and

$$
\begin{aligned}
& b_{1}=(1-p) \beta_{0} \\
& b_{2}=4(p-1)(n-4) \beta_{1} \\
& b_{3}=2(n-4)(n+2 p-2) \beta_{1} \\
& b_{4}=-4(n-4)(n+2 p-5) \beta_{1} \\
& b_{5}=(n-6)(n+2 p-4) \beta_{1}
\end{aligned}
$$

is a second-order conformal covariant with $\omega=-2$ and $4 \omega_{0}=4+2 p-n$.

Proof. The operator $\stackrel{s}{D_{(2, p)}^{*}}$ is a linear combination of the Weyl curvature terms of $\stackrel{s}{D}(4, p)$ and the term $T S\left[C_{i_{1} \ldots}^{a b d} \stackrel{c}{\nabla} \stackrel{c}{a}^{c} u_{d i_{2} \ldots i_{p}}\right]$. Using $4 \omega_{0}+n-2 p-4=0$ we obtain

$$
\begin{aligned}
X^{\gamma}\left(C_{i_{1} \ldots d}^{a b d} \nabla_{a}^{c}{ }^{c}{ }_{b} u_{d i_{2} \ldots i_{p}}\right) \stackrel{*}{=} & (p-1)\left[\delta_{i_{1}}^{\gamma} C_{i_{2} \ldots d}^{a b}{ }^{c}{ }_{d} u_{a b i_{3} \ldots i_{p}}-C_{. i_{1} i_{2} .}^{a}{ }^{b}{ }_{a}^{c} u_{. b i_{3} \ldots i_{p}}^{\gamma}\right] \\
& +(n-3) C_{i_{1} \ldots}^{a b \gamma} \stackrel{c}{\nabla}_{a} u_{b i_{2} \ldots i_{p}}-\frac{n}{2} C_{i_{1} \ldots}^{a b \gamma}{ }^{c} \nabla_{b} u_{. a i_{2} \ldots i_{p}}
\end{aligned}
$$

and after lengthy straight computations one verifies the result $X^{\gamma} \stackrel{s}{D_{(2, p)}^{*}}=0$ 
Remark 5.2. The operator $\stackrel{s}{D_{(2,1)}^{*}}$ has been found already by Graham [24]. Only if $n=4$ and $p=1$, Bach tensor expressions obstruct the existence of $\stackrel{s}{D}(4,1)$ and $\stackrel{s}{D_{(2,1)}^{*}}$. However, by means of $\stackrel{s}{D}_{(2,1)}^{*}$ one can substitute the Bach tensor expression of $\stackrel{s}{D}(4,1)$ such that one obtains a conformal covariant also in the critical case $n=4$ and $p=1$ by a suitable linear combination of $\stackrel{s}{D}_{(4,1)}$ and $\stackrel{s}{D}_{(2,1)}^{*}$ (see also [24]).

Lemma 5.1. The operators $T S\left[\stackrel{c}{\nabla}_{i_{1}} \ldots \stackrel{c}{\nabla}_{i_{k}} u_{i_{k+1} \ldots i_{k+p}}\right]$ are conformal covariants on $\stackrel{s}{\mathcal{T}_{p}}$ with $\omega=0$ and $2 \omega_{0}=2 p+k-1$.

The proof is a direct consequence of (see [42])

$$
X^{\gamma} T S\left[\stackrel{c}{\nabla}_{i_{1}} \ldots \stackrel{c}{\nabla}_{i_{k}} u_{i_{k+1} \ldots i_{k+p}}\right]=k\left[2 \omega_{0}-2 p-k+1\right] T S\left[\delta_{i_{1}}^{\gamma} \stackrel{c}{\nabla}_{i_{2}} \ldots \stackrel{c}{\nabla}_{i_{k}} u_{i_{k+1} \ldots i_{k+p}}\right] .
$$

Corollary 5.1. A symmetric, trace-free tensor $E_{i_{1} \ldots i_{k+p}}$ from $\vartheta_{0}$ is conformally invariant if and only if the operator

$$
E^{i_{1} \ldots i_{k+p}} \stackrel{c}{\nabla} i_{i_{1}} \ldots \stackrel{c}{\nabla_{i_{k}}} u_{i_{k+1} \ldots i_{k+p}}
$$

is a conformal covariant with $2 \omega_{0}=2 p+k-1$.

Proof. The first part is a consequence of Lemma 5.1. Conversely, since the derivatives of $u_{i_{1} \ldots i_{p}}$ at any fixed point can be chosen arbitrarily, the assertion $X^{\gamma} E^{i_{1} \ldots i_{k+p}}=0$ follows from the conformal invariance of (5.2)

Corollary 5.2. The nonlinear operators $T_{(k, p)}$ defined on $\mathcal{T}_{p}$ by

$$
T_{(k, p)}[u]:=\left(T S\left[\stackrel{c}{\nabla}^{i_{1}} \ldots{\stackrel{c}{\nabla} i^{i_{k}}}^{i_{k+1} \ldots i_{k+p}}\right]\right)\left[\stackrel{c}{\nabla}_{i_{1}} \ldots \stackrel{c}{\nabla}_{i_{k}} u_{i_{k+1} \ldots i_{k+p}}\right]
$$

are conformal covariants with $2 \omega_{0}=2 p+k-1$.

\section{Conformal covariants acting on differential forms}

Let $\Lambda^{p}(p \geq 1)$ denote the space of all $p$-forms of class $C^{\infty}$. When $\alpha_{1}, \ldots, \alpha_{p}$ appear in the sequel, we assume that alternation has been carried out over these indices. Branson found in [5] a second-order conformal covariant $D_{2, p}$ on $\Lambda^{p}$ with $\omega=-1,4 \omega_{0}=2+2 p-n$ and a fourth-order conformal covariant $D_{4, p}$ on $\Lambda^{p}$ with $\omega=-2,4 \omega_{0}=4+2 p-n(n>$ 4). The operator $D_{4, p}$ differs only by a real factor to the conformal covariant $D_{(4, p)}$ constructed by the present author in [40] by means of the method given in Section 2 . We have $D_{(4,1)}=-\frac{n+2}{4} \stackrel{s}{D}_{(4,1)}$ (see Theorem 5.2).

As for $D_{(6)}$ and $\stackrel{s}{D}(4,1)$, a Bach tensor expression of the form

$$
\frac{c(n, p)}{n-4} B_{\alpha_{1}}{ }^{k} u_{k \alpha_{2} \ldots \alpha_{p}} \quad \text { with } c(n, p) \in \mathbb{R} \backslash\{0\}
$$

obstructs the existence of $D_{(4, p)}$ in dimension 4 (if $p=1$, see Remark 5.2). 
Problem C. Is there a fourth-order conformal covariant on $\Lambda^{2}$ with the leading term $\square^{2}$ in dimension 4 ?

The proof of the following theorem is analogous to the proofs of Theorems 5.2 and 5.3 .

Theorem 6.1. Suppose that $n>4,2 p \neq n+6$ or $p=2, n=4$. Let $D_{(2, p)}^{*}$ be the operator defined on $\Lambda^{p}$ by

$$
\begin{aligned}
& \left(D_{(2, p)}^{*}[u]\right)_{\alpha_{1} \ldots \alpha_{p}}:=C_{\alpha_{1} \ldots}^{a b d}\left(\stackrel{c}{\nabla} \stackrel{c}{a}_{b} u_{d \alpha_{2} \ldots \alpha_{p}}+2 c_{1} \stackrel{c}{\nabla}{ }_{\alpha_{2}} \stackrel{c}{\nabla} a u_{b d \alpha_{3} \ldots \alpha_{p}}\right) \\
& +c_{1} C_{\alpha_{1} \alpha_{2} \ldots}^{a b}\left(\stackrel{c}{\square} u_{a b \alpha_{3} \ldots \alpha_{p}}+2 \stackrel{c}{\nabla}_{a} \stackrel{c}{\nabla}^{k} u_{k b \alpha_{3} \ldots \alpha_{p}}\right) \\
& -4 c_{1} \stackrel{c}{\nabla}{ }_{\alpha_{1}} C_{\alpha_{2} \ldots}^{a b d} \stackrel{c}{\nabla}_{a} u_{b d \alpha_{3} \ldots \alpha_{p}}+c_{2} \stackrel{c}{\nabla}_{\alpha_{1}}\left(\stackrel{c}{\nabla}_{u} C_{. \alpha_{2} . .}^{u a b} u_{a b \alpha_{3} \ldots \alpha_{p}}\right) \\
& +c_{3}\left(\stackrel{c}{\nabla}_{u} C_{. \alpha_{2} . .}^{u}{ }^{a b} \stackrel{c}{\nabla}_{\alpha_{2}} u_{a b \alpha_{3} \ldots \alpha_{p}}+\stackrel{c}{\nabla}_{u} C_{\ldots \alpha_{1} \alpha_{2}}^{u a} \stackrel{c}{\nabla}^{k} u_{k a \alpha_{2} \ldots \alpha_{p}}\right) \\
& +\stackrel{c}{\nabla}{ }_{u} C_{\ldots \alpha_{1}}^{u a b}\left(c_{4} \stackrel{c}{\nabla}_{b} u_{a \alpha_{2} \ldots \alpha_{p}}+c_{5} \stackrel{c}{\nabla}_{a} u_{b \alpha_{2} \ldots \alpha_{p}}\right) \\
& +c_{6} B_{\alpha_{1} .}^{a} u_{a \alpha_{2} \ldots \alpha_{p}}+c_{7} C_{\alpha_{1} \alpha_{2}}^{a b} \stackrel{c}{\nabla}_{\alpha_{3}} \stackrel{c}{\nabla}^{k} u_{k a b \alpha_{4} \ldots \alpha_{p}}
\end{aligned}
$$

where

$$
\left.\begin{array}{rl}
c_{1} & =\frac{3(p-1)}{2(n-2 p+6)} \\
c_{2} & =\frac{2(n-2)}{n-3} c_{1} \\
c_{3} & =\frac{n}{n-3} c_{1} \\
c_{4} & =\frac{6(1-p)+(3-n)(n-2 p+6)}{(n-3)(n-2 p+6)} \\
c_{5} & =\frac{n}{2(n-3)} \\
c_{6} & =\frac{(n-2)[12(p-1)+(n-6)(n-2 p+6)]}{4(n-3)(n-4)(n-2 p+6)} \\
c_{7} & =0 .
\end{array}\right\}
$$

Then $D_{(2, p)}^{*}$ is a second-order conformal covariant with $\omega=-2$ and $4 \omega_{0}=4+2 p-n$.

Remark 6.1. As expected there holds $D_{(2,1)}^{*}=\stackrel{s}{D_{(2,1)}^{*}}$. As already noted, one can annihilate the obstructing Bach tensor expression of $D_{(4,1)}$ in dimension 4 by means of $\stackrel{s}{D_{(2,1)}^{*}}$. If $p=3$, then we have an analogous situation. If $p=2$, we have $c_{6}=$ $\frac{n(n-2)}{4(n-3)(n+2)}$. Hence, the operator $D_{(2,2)}^{*}$ is a regular operator in the important case $p=2$ and $n=4$. Consequently, the obstructing Bach tensor expression (6.1) of $D_{(4,2)}$ cannot be substituted by $D_{(2,2)}^{*}$.

Theorem 6.2. If $n=4$, there is no conformal covariant on $\Lambda^{2}$ with leading term $\square^{2}$. 
Proof. Under consideration of (3.2) and (3.3), it is easy to see that the operator $D_{(2, p)}^{*}$ contains all possible curvature monomials if $\omega=-2$ and $4 \omega_{0}=4+2 p-n$ (modulo trivial order zero actions of the Weyl tensor). Furthermore, the real coefficients of $D_{(2,1)}^{*}$ and $D_{(4, p)}$ (see [40]: p. 279]) are determined uniquely. Now the fact that the obstructing Bach tensor expression (6.1) of $D_{(4,2)}$ cannot be substituted by $D_{(2,2)}^{*}$ implies the assertion

\section{References}

[1] Bach, R.: Zur Weylschen Relativitätstheorie. Math. Z. 9 (1921), 110 - 135.

[2] Bailey, T. N. and M. G. Eastwood: Complex paraconformal manifolds - their differential geometry and twistor theory. Forum Math. 3 (1991), 61 - 103.

[3] Baston, J. R.: Verma modules and differential conformal invariants. J. Diff. Geom. 32 (1990), $851-898$.

[4] Branson, T.: Conformally covariant equations on differential forms. Comm. in P.D.E. 7 (1982), 393-431.

[5] Branson, T.: Differential operators canonically associated to a conformal structure. Math. Scand. 57 (1985), $293-345$.

[6] Branson, T.: Second-order conformal covariants. Preprint. Copenhagen: University preprint series, Nr. 2 and 3 (1989).

[7] Branson, T.: Conformal transformations, conformal change and conformal covariants. Supp. Rend. Cir. Mat. Palermo (II) 21 (1989), 115 - 134.

[8] Branson, T.: Nonlinear Phenomena in the spectral theory of geometric linear differential operators. In: Proc. Symposia Pure Math. 59 (1996), 28 - 65.

[9] Branson, T., Olafsson, G. and B. Ørsted: Spectrum generating operators, and intertwining operators for representations intuced from a maximal parabolic subgroup. Funct. Anal. (to appear).

[10] Branson, T. and B. Ørsted: A conformal index for Riemannian manifolds. Preprint 1985.

[11] Branson, T. and B. Ørsted: Conformal indices of Riemannian manifolds. Compositio Math. 60 (1986), $261-293$.

[12] Branson, T. and B. Ørsted: Conformal geometry and global invariants. Diff. Geom. Appl. 1 (1991), $279-308$.

[13] Carminati, J. and G. Mclenaghan: An explicit determination of the space-times on which the conformally invariant scalar wave equation satisfy Huygens' principle. Ann. Inst. Henri Poincar, Phys. Théor. 44 (1986), 115 - 153; Part. II: 47 (1987), 337 - 354; Part. III: 48 (1988), $77-96$ and 54 (1991), $9-16$.

[14] du Plessis, J. C.: Polynomial conformal tensors. Proc. Camb. Phil. Soc. 68 (1970), 329 -344 .

[15] du Plessis, J. C.: Conformal concomitants and continuity equations. Tensor (N.S.) 21 (1970), $1-14$.

[16] Eastwood, M. and J. Rice: Conformally invariant differential operators on Minkowski spasce and their curved analogues. Comm. Math. Phys. 109 (1987), $207-228$.

[17] Epstein, D.: Natural tensors on Riemannian manifolds. J. Diff. Geom. 10 (1975), 631 645. 
[18] Erdmenger, J.: Conformally covariant differential operators: properties and applications. Class. Quantum Grav. 14 (1997), 2061 - 2084.

[19] Fefferman, C. and C. R. Graham: Conformal invariants. Èliie Cartan et les Mathématiques d' Aujourdhui, Astrisque (1985), 95 - 116.

[20] Fegan, H. D.: Conformally invariant first order differential operators. Quart. J. Math. Oxford 27 (1976), $371-378$.

[21] Gerlach, R. and V. Wüsch: Contributions to polynomial conformal tensors. Ann. Inst. Henri Poincaré, Physique théorique 70 (1999), 313 - 340.

[22] Gover, R.: Conformally invariant operators of standard type. Quart. J. Math. Oxford (2) 40 (1989), 197 - 207.

[23] Graham, C. R., Jenne, R. L., Mason, J. and G. A. J. Sparling: Conformally invariant powers of the Laplacian. Part I: Existence. J. Lond. Math. Soc. 46 (1992), 557 - 565.

[24] Graham, C. R.: Conformally invariant powers of the Laplacian. Part I: Nonexistence. J. Lond. Math. Soc. 46 (1992), $566-576$.

[25] Günther, P.: Huygens' Principle and Hyperbolic Equations. Boston: Acad. Press 1988.

[26] Günther, P.: A class of conformally invariant symmetric tensors of weight zero. Math. Nachr. 144 (1989), 149 - 164.

[27] Günther, P. and V. Wünsch: Contributions to a theory of polynomial conformal tensors. Math.Nachr. 126 (1986), 83 - 100.

[28] Günther, P. and V. Wünsch: On some polynomial conformal tensors. Math. Nachr. 124 (1985), $217-238$.

[29] Hesselbach, B.: Über die Erhaltungssätze der konformen Geometrie. Math. Nachr. 3 (1949), $107-126$.

[30] Mclenagan, R. G.: Huygens' Principle. Ann. Inst. Henri Poincaré A 37 (1982), 211 236.

[31] Ørsted, B.: Conformally invariant differential equations and projective geometry. J. Funct. Anal. 44 (1981), 1 - 23.

[32] Ørsted, B.: The conformal invariance of Huygens' principle. J. Diff. Geom. 16 (1981), 1 $-9$.

[33] Paneitz, S.: A quartic conformally covariant differential operator for arbitrary pseudoRiemannian manifolds. Preprint 1983.

[34] Schimming, R.: Konforminvarianten vom Gewicht -1 eines Zusammenhanges oder Eichfeldes. Z. Anal. Anw. 3 (1984), $401-412$.

[35] Schimming, R.: Cauchy-Problem und Wellenlösungen der Bachschen Feldgleichungen der Allgemeinen Relativitätstheorie. Habilitationsschrift. Leipzig: Universität 1979.

[36] Stellmacher, K. L.: Geometrische Deutung konforminvarianter Eigenschaften des Riemannschen Raumes, Math. Ann. 123 (1951), 34-52.

[37] Wünsch, V.: Über eine Klasse konforminvarianter Tensoren. Math. Nachr. 73 (1976), $37-58$.

[38] Wünsch, V.: Cauchy-Problem und Huygenssches Prinzip bei einigen Klassen spinorieller Feldgleichungen, Parts I and II. Beitr. zur Analysis 12 (1978), 47 - 76 and 12 (1979), 147 $-177$.

[39] Wünsch, V.: Konforminvariante Variationsprobleme und Huygenssches Prinzip. Math. Nachr.120 (1985), 175 - 193. 
[40] Wünsch, V.: On conformally invariant differential operators. Math. Nachr. 129 (1986), $269-281$.

[41] Wünsch, V.: Conformal C- and Einstein Spaces. Math. Nachr. 146 (1990), 237 - 245.

[42] Wünsch, V.: Moments and Huygens' principle for conformally invariant field equations in curved space-times. Ann. Inst. Henri Poincaré, Physique théorique. 60 (1994), 433 455.

[43] Yamabe, H.: On a deformation of Riemannian structures on compact manifolds. Osaka J. Math. 12 (1960), $21-37$.

Received 04.05.1999 